\title{
Title: 6-Thioguanine blocks SARS-CoV-2 replication by inhibition of PLpro
}

\section{protease activities}

Authors: Caleb D. Swaim ${ }^{1}$, Yi-Chieh Perng ${ }^{2}$, Xu Zhao ${ }^{1}$, Larissa A. Canadeo ${ }^{1}$, Houda H.

Harastani ${ }^{2}$, Tamarand L. Darling ${ }^{2}$, Adrianus C. M. Boon ${ }^{2,3,4}$, Deborah J. Lenschow ${ }^{2,3}$, and Jon M. Huibregtse ${ }^{1 *}$.

Affiliations: ${ }^{1}$ Department of Molecular Biosciences, University of Texas at Austin, Austin, TX,

${ }^{2}$ Department of Internal Medicine, ${ }^{3}$ Department of Pathology and Immunology, ${ }^{4}$ Department of Molecular Microbiology, Washington University School of Medicine, St. Louis, MO.

*Correspondence to: huibregtse@austin.utexas.edu.

One Sentence Summary: A repurposed drug that targets an essential enzymatic activity of SARS-CoV-2 represents a promising COVID-19 therapeutic.

Abstract: A recently emerged betacoronavirus, SARS-CoV-2, has led to a global health crisis that calls for the identification of effective therapeutics for COVID-19 disease. Coronavirus papain-like protease (PLpro) is an attractive drug target as it is essential for viral polyprotein cleavage and for deconjugation of ISG15, an antiviral ubiquitin-like protein. We show here that 6-Thioguanine (6-TG) inhibits SARS-CoV-2 PLpro-catalyzed viral polyprotein cleavage and ISG15 deconjugation in cells and inhibits replication of SARS-CoV-2 in Vero-E6 cells and Calu3 cells at submicromolar levels. As a well-characterized FDA-approved orally delivered drug, 6-TG represents a promising therapeutic for COVID-19 and other emerging coronaviruses. 


\section{Main Text:}

Coronavirus Disease-2019 (COVID-19) is caused by SARS-CoV-2, a betacoronavirus highly similar to SARS (now SARS-CoV-1) $(1,2)$. An urgent need exists for treatment strategies, and repurposed FDA-approved drugs with existing supply chains and well-characterized pharmacologic properties represent a rapid and efficient approach toward COVID-19 therapeutics (3).

Enzymes encoded by SARS-CoV-2 are attractive drug targets, including PLpro, an essential cysteine protease activity within the nsp3 protein (4). PLpro cleaves the ppla polyprotein at three sites, generating mature nsp1, 2 and 3 proteins. The PLpro recognition site (LXGG) is also found at the C-terminus of ubiquitin and ISG15, an antiviral ubiquitin-like modifier, although SARS-CoV-1 and -2 PLpro preferentially catalyze de-ISGylation over de-ubiquitylation $(5,6)$. Therapeutic inhibition of PLpro would therefore be predicted to have two antiviral effects: restoration of the antiviral effect of ISGylation and inhibition of viral replication by blocking polyprotein cleavage. Further, de-ISGylation by PLpro, through generation of free (unconjugated) ISG15, enhances the secretion and extracellular signaling function of ISG15, which in turn promotes pro-inflammatory cytokine production from cells of the immune system (7). Therefore, a potential third effect of inhibiting PLpro would be a decrease in pro-inflammatory "cytokine storms" associated with COVID-19 (8).

6-Thioguanine (6-TG) is an FDA-approved drug that has been used in the clinic since the 1950s, originally for the treatment of childhood leukemias and subsequently for treatment of inflammatory bowel and Crohn's disease (9). 6-TG was previously reported to inhibit the SARS-CoV-1 and MERS PLpro catalytic domain in vitro $(10,11)$, with an IC50 of $5 \mu \mathrm{M}$ and 
$24.4 \mu \mathrm{M}$, respectively, however there was no further follow up of its ability to inhibit de-ISGylation or viral polyprotein cleavage in cells or its ability to inhibit viral replication. Fig. 1A shows that co-expression of PLpro (residues 746-1061 of nsp3) with ISG15 and its conjugation enzymes (Uba7, Ube2L6, and Herc5) in HEK293T cells resulted in nearly complete loss of ISG15 conjugates, while expression of the active-site C-to-A variant of PLpro (C856 of nsp3; PLpro ${ }^{\mathrm{CA}}$ ) was inactive. Addition of 6-TG resulted in a dose-dependent inhibition of PLpro de-ISGylation activity, with half-maximal inhibition at approximately $0.1 \mu \mathrm{M}$ (Fig. 1A; quantitation shown in Supplementary Fig. 1A).

The PLpro domain, located within nsp3, generates mature nsp-1, 2 and 3 proteins from the ppla polyprotein through self-catalyzed cleavage reactions (Fig. 1B, schematic). We determined whether 6-TG inhibited polyprotein processing, as this is an essential function of Plpro and the natural context in which PLpro is expressed. An N-terminally TAP-tagged fusion of nsp1, 2 and 3 (TAP-nsp123 ${ }^{\text {WT }}$ ) was expressed in HEK293T cells (Fig. 1B, schematic), as well as the active-site C-to-A variant (TAP-nsp123 ${ }^{\mathrm{CA}}$ ). The fusion protein was detected with anti-TAP antibody, and TAP-nsp1 was expressed separately as a size marker for the fully processed fusion protein. As shown in Fig. 1B, expression of TAP-nsp $123^{\mathrm{WT}}$ resulted primarily in a product the size of TAP-nsp1 (42 kD), as expected if the fusion protein was fully processed, while expression of TAP-nsp $123^{\mathrm{CA}}$ resulted in the expected full-length protein $(\sim 336 \mathrm{kD})$ along with several breakdown products of the full-length protein. Increasing concentrations of 6-TG inhibited PLpro-mediated processing of the TAP-nsp123 ${ }^{\mathrm{WT}}$ polyprotein, with loss of the TAP-nsp1 product and accumulation of the full-length fusion protein, with the same pattern of breakdown products seen with the TAP-nsp $123^{\mathrm{CA}}$ protein. Half-maximal inhibition of 
polyprotein cleavage occurred between 0.1 and $0.5 \mu \mathrm{M}$ (quantitation shown in Supplementary

Fig. 1B). This result indicates that 6-TG inhibits an essential function of PLpro activity and that it inhibits PLpro when it is expressed in the context of the full-length nsp3 protein.

In addition to its function as a ubiquitin-like modifier, free (unconjugated) ISG15 exits cells and signals to LFA-1-expressing cells of the immune system (e.g., NK cells, T cells) to release specific cytokines, including IFN- $\gamma$ and IL-10 $(7,12)$, while ISG15 conjugates are retained in cells. Therefore, a secondary effect of PLpro-mediated de-ISGylation is the enhanced secretion of ISG15 (7). We determined whether 6-TG would block this effect. Fig. 2A shows that expression of PLpro in HEK293T cells led to decreased intracellular ISG15 conjugates (upper blot, total cell lysates) and increased free ISG15 in the extracellular space (lower blot, IP-western of ISG15 from cell culture supernatants), and that 6-TG not only restored intracellular conjugates but also blocked the production of free extracellular ISG15.

IFN- $\gamma$, IL-10 and IL-6 are associated with uncontrolled cytokine release syndrome and have been shown to be elevated in COVID-19 patients $(13,14)$. The complete range of cytokines affected by extracellular ISG15 signaling is unknown, but the relationship of ISG15 to IFN- $\gamma$ and IL-10 (12) led us to test whether ISG15 also induced the secretion of IL-6 from human PBMCs. In a trans-well culture system, HEK293T cells (lower chamber) were transfected with ISG15, with or without the ISG15 conjugation enzymes (E1/E2/E3) and/or PLpro. PBMCs were placed in the upper chamber, separated from the lower chamber by a 0.4 micron membrane. In this system, ISG15 released from cells in the lower chamber signals to LFA-1-expressing cells in the upper chamber, and cytokine release in the upper chamber is measured by ELISA (7). Fig. 2B shows that expression of ISG15, alone, in the HEK293T cells led to secretion of IL-6 from 
PBMCs, and this effect was diminished when the conjugation enzymes were co-expressed with ISG15 (compare lanes 1, 3, and 5). De-ISGylation catalyzed by PLpro resulted in enhanced IL-6 secretion (compare lanes 5 and 7), and the addition of 6-TG reversed the effect of PLpro, leading to diminished IL-6 production (compare lanes 7 and 8). These results identify IL-6 as an additional cytokine that is responsive to extracellular ISG15 signaling and suggest that PLpro may be at least partially responsible for enhanced cytokine responses in COVID-19 patients. Therapeutically, 6-TG inhibition of PLpro might therefore have the additional benefit of limiting excessive cytokine responses associated with poor patient outcomes.

6-TG was next analyzed for its ability to inhibit SARS-CoV-2 replication (strain 2019 n-CoV/USA_WA1/2020; 18) in Vero-E6 African green monkey kidney cells and in the Calu3 human lung epithelial cell line. As shown in Fig. 3, 6-TG inhibited viral replication in Vero-E6 cells with a half-maximal effective concentration (EC50) of $0.647 \pm 0.374 \mu \mathrm{M}$. By comparison, Remdesivir inhibited SARS-CoV-2 replication in Vero-E6 cells similarly, with an EC50 of 0.77 $\mu \mathrm{M}$ (15). 6-TG inhibited virus replication in Calu3 cells at a lower EC50, $0.061 \pm 0.049 \mu \mathrm{M}$. While the basis of the difference between the two cell lines is not known, cell lines can vary significantly with respect to thiopurine uptake and metabolism (16). 6-TG did not elicit significant cellular toxicity in either Vero-E6 or Calu3 cells (CC50>50 $\mu \mathrm{M})$, yielding selectivity indices (SI) of $>77$ in Vero-E6 cells and $>819$ Calu3 cells. Together, the results presented here indicate that 6-Thioguanine is a direct-acting SARS-CoV-2 antiviral, inhibiting PLpro de-ISGylation, polyprotein cleavage, and viral replication at submicromolar concentrations.

To our knowledge, 6-TG is the first identified FDA-approved inhibitor of PLpro demonstrated to inhibit SARS-CoV-2 replication. 6-TG is a widely available orally delivered 
generic drug on the World Health Organization list of essential medicines (17). Dosing regimens vary significantly depending on its use, from $10 \mathrm{mg}$ per day for long-term treatment of inflammatory diseases, to up to $3 \mathrm{mg} / \mathrm{kg} /$ day in acute lymphocytic leukemia treatments (18). While toxicity can be significant at higher dosages, it is anticipated that its use as an antiviral would be over a relatively short time period and that toxicity issues would likely be minimal. We propose that the results presented here warrant the initiation of human clinical trials of 6-TG as a SARS-CoV-2 therapeutic. As PLpro is a conserved and essential enzymatic activity of the beta coronaviruses, 6-TG may prove useful in the current and future coronavirus pandemics and as a complement to other antivirals in development. 


\section{References and Notes:}

1. L. E. Gralinski, V. D. Menachery, Return of the Coronavirus: 2019-nCoV. Viruses. 12 (2020), doi:10.3390/v12020135.

2. Coronaviridae Study Group of the International Committee on Taxonomy of Viruses, The species Severe acute respiratory syndrome-related coronavirus: classifying 2019-nCoV and naming it SARS-CoV-2. Nat Microbiol. 5, 536-544 (2020).

3. R. K. Guy, R. S. DiPaola, F. Romanelli, R. E. Dutch, Rapid repurposing of drugs for COVID-19. Science. 368, 829-830 (2020).

4. B. H. Harcourt, D. Jukneliene, A. Kanjanahaluethai, J. Bechill, K. M. Severson, C. M. Smith, P. A. Rota, S. C. Baker, Identification of severe acute respiratory syndrome coronavirus replicase products and characterization of papain-like protease activity. J. Virol. 78, 13600-13612 (2004).

5. C. M. Daczkowski, J. V. Dzimianski, J. R. Clasman, O. Goodwin, A. D. Mesecar, S. D. Pegan, Structural Insights into the Interaction of Coronavirus Papain-Like Proteases and Interferon-Stimulated Gene Product 15 from Different Species. J. Mol. Biol. 429, 1661-1683 (2017).

6. B. T. Freitas, I. A. Durie, J. Murray, J. E. Longo, H. C. Miller, D. Crich, R. J. Hogan, R. A. Tripp, S. D. Pegan, Characterization and noncovalent inhibition of the deubiquitinase and deISGylase activity of SARS-CoV-2 papain-like protease. ACS Infect Dis (2020), doi:10.1021/acsinfecdis.0c00168.

7. C. D. Swaim, L. A. Canadeo, K. J. Monte, S. Khanna, D. J. Lenschow, J. M. Huibregtse, Modulation of Extracellular ISG15 Signaling by Pathogens and Viral Effector Proteins. Cell Reports. 31 (2020), doi:10.1016/j.celrep.2020.107772.

8. M. Z. Tay, C. M. Poh, L. Rénia, P. A. MacAry, L. F. P. Ng, The trinity of COVID-19: immunity, inflammation and intervention. Nat. Rev. Immunol. (2020), doi:10.1038/s41577-020-0311-8.

9. A. B. Bayoumy, M. Simsek, M. L. Seinen, C. J. J. Mulder, A. Ansari, G. J. Peters, N. K. De Boer, The continuous rediscovery and the benefit-risk ratio of thioguanine, a comprehensive review. Expert Opin Drug Metab Toxicol. 16, 111-123 (2020).

10. K.-W. Cheng, S.-C. Cheng, W.-Y. Chen, M.-H. Lin, S.-J. Chuang, I.-H. Cheng, C.-Y. Sun, C.-Y. Chou, Thiopurine analogs and mycophenolic acid synergistically inhibit the papain-like protease of Middle East respiratory syndrome coronavirus. Antiviral Res. 115, 9-16 (2015).

11. C.-Y. Chou, C.-H. Chien, Y.-S. Han, M. T. Prebanda, H.-P. Hsieh, B. Turk, G.-G. Chang, X. Chen, Thiopurine analogues inhibit papain-like protease of severe acute respiratory syndrome coronavirus. Biochem. Pharmacol. 75, 1601-1609 (2008).

12. C. D. Swaim, A. F. Scott, L. A. Canadeo, J. M. Huibregtse, Extracellular ISG15 Signals Cytokine Secretion through the LFA-1 Integrin Receptor. Mol. Cell. 68, 581-590.e5 (2017).

13. C. Huang, Y. Wang, X. Li, L. Ren, J. Zhao, Y. Hu, L. Zhang, G. Fan, J. Xu, X. Gu, Z. Cheng, T. Yu, J. Xia, Y. Wei, W. Wu, X. Xie, W. Yin, H. Li, M. Liu, Y. Xiao, H. Gao, L. Guo, J. Xie, G. Wang, R. Jiang, Z. Gao, Q. Jin, J. Wang, B. Cao, Clinical features of patients infected with 2019 novel coronavirus in Wuhan, China. The Lancet. 395, 497-506 (2020).

14. D. McGonagle, K. Sharif, A. O’Regan, C. Bridgewood, The Role of Cytokines including Interleukin-6 in COVID-19 induced Pneumonia and Macrophage Activation Syndrome-Like Disease. Autoimmun Rev. 19, 102537 (2020).

15. M. Wang, R. Cao, L. Zhang, X. Yang, J. Liu, M. Xu, Z. Shi, Z. Hu, W. Zhong, G. Xiao, Remdesivir and chloroquine effectively inhibit the recently emerged novel coronavirus (2019-nCoV) in vitro. Cell Res. 30, 269-271 (2020).

16. J. A. Duley, A. A. Somogyi, J. H. Martin, The future of thiopurine pharmacogenomics. Pharmacogenomics. 13, 1549-1552 (2012).

17. World Health Organizaiton Model List of Essential Medicines, (available at https://apps.who.int/iris/bitstream/handle/10665/325771/WHO-MVP-EMP-IAU-2019.06-eng.pdf).

18. M. L. Seinen, D. P. van Asseldonk, C. J. J. Mulder, N. K. H. de Boer, Dosing 6-thioguanine in inflammatory bowel disease: expert-based guidelines for daily practice. J Gastrointestin Liver Dis. 19, 291-294 (2010). 
Acknowledgments: We thank Robert Krug and Sylvie Beaudenon-Huibregtse for critical reading of the manuscript and Bill Matsui for helpful discussions. Funding: This work was supported by grants from the National Institutes of Health, National Institute of Allergy and Infectious Disease to J. M. H. (AI096090) and D. J. L. (AI080672) and a CTSA from the National Institutes of Health (UL1 TR002345) to D. J. L. Author contributions: Conceptualization and methodology: J. M. H., D. J. L., C. D. S., Y.-C. P., L. A. C.; Investigation and data analysis: C. D. S., Y.-C. P., X. Z., H. H. H., T. L. D.; Supervision: J. M. H., D. J. L., A. C. M. B.; Writing, original draft: J. M. H.; Writing, editing: D. J. L., A. C. M. B., C. D. S., Y.-C. P., L. A. C.; Project administration and funding acquisition: J. M. H., D. J. L., A. C. M. B. Competing interests: Authors declare no competing interests. Data and materials availability: All materials that are not commercially available or available from public resources are available from the corresponding author. All data is available in the main text or the supplementary materials.

\section{List of Supplementary Materials:}

Materials and Methods

Figures S1 
A

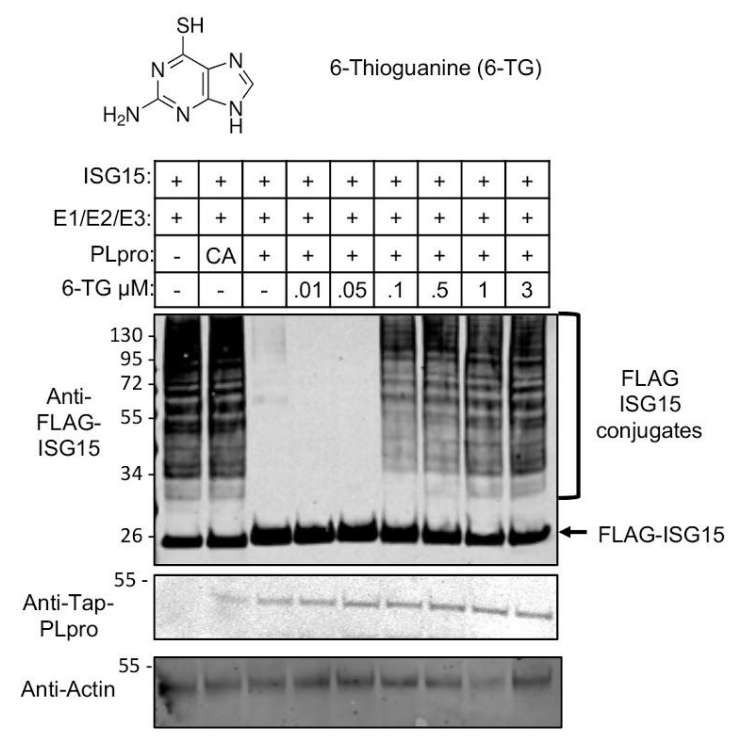

B
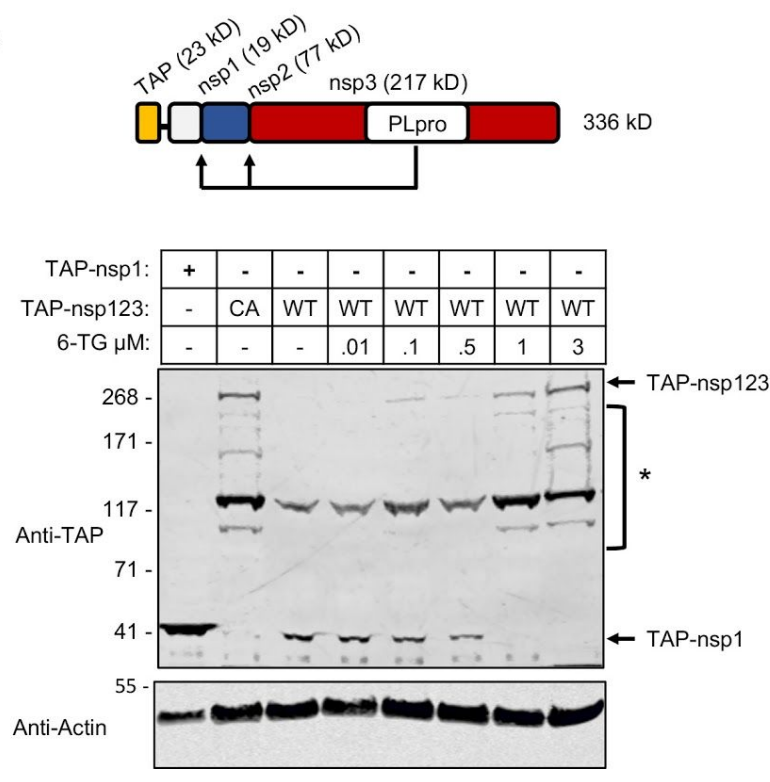

Figure 1. 6-TG inhibits PLpro mediated de-ISGylation and viral polyprotein cleavage. A. The structure of 6-Thioguanine is shown. HEK293T cells were transfected with plasmids expressing FLAG-ISG15 and the ISG15 E1/E2/E3 enzymes and $0.2 \mu \mathrm{g}$ of PLpro plasmid (WT or CA) where indicated. 6-TG was added at the time of transfection at the indicated final concentrations $(\mu \mathrm{M})$. Total cell lysates were prepared 48 hours post-transfection and analyzed by immunoblotting to detect FLAG-ISG15 and FLAG-ISG15 conjugates. B. (Top) Schematic representation of the TAP-nsp123 fusion protein, with molecular masses of subdomains indicated. Arrows indicate sites of PLpro-catalyzed cleavage. (Bottom) HEK293T cells were transfected with plasmids expressing either TAP-nsp1, TAP-nsp $123^{\mathrm{WT}}$ or TAP-nsp123 ${ }^{\mathrm{CA}}$. 6-TG was added at the time of transfection at the indicated final concentrations $(\mu \mathrm{M})$ and total cell lysates were prepared 48 hours post-transfection and analyzed by immunoblotting with anti-TAP antibody. Bands corresponding to TAP-nsp1 and the full-length fusion proteins are indicated. Bracketed bands $\left(^{*}\right)$ represent breakdown products of the full-length WT or CA fusion proteins. 

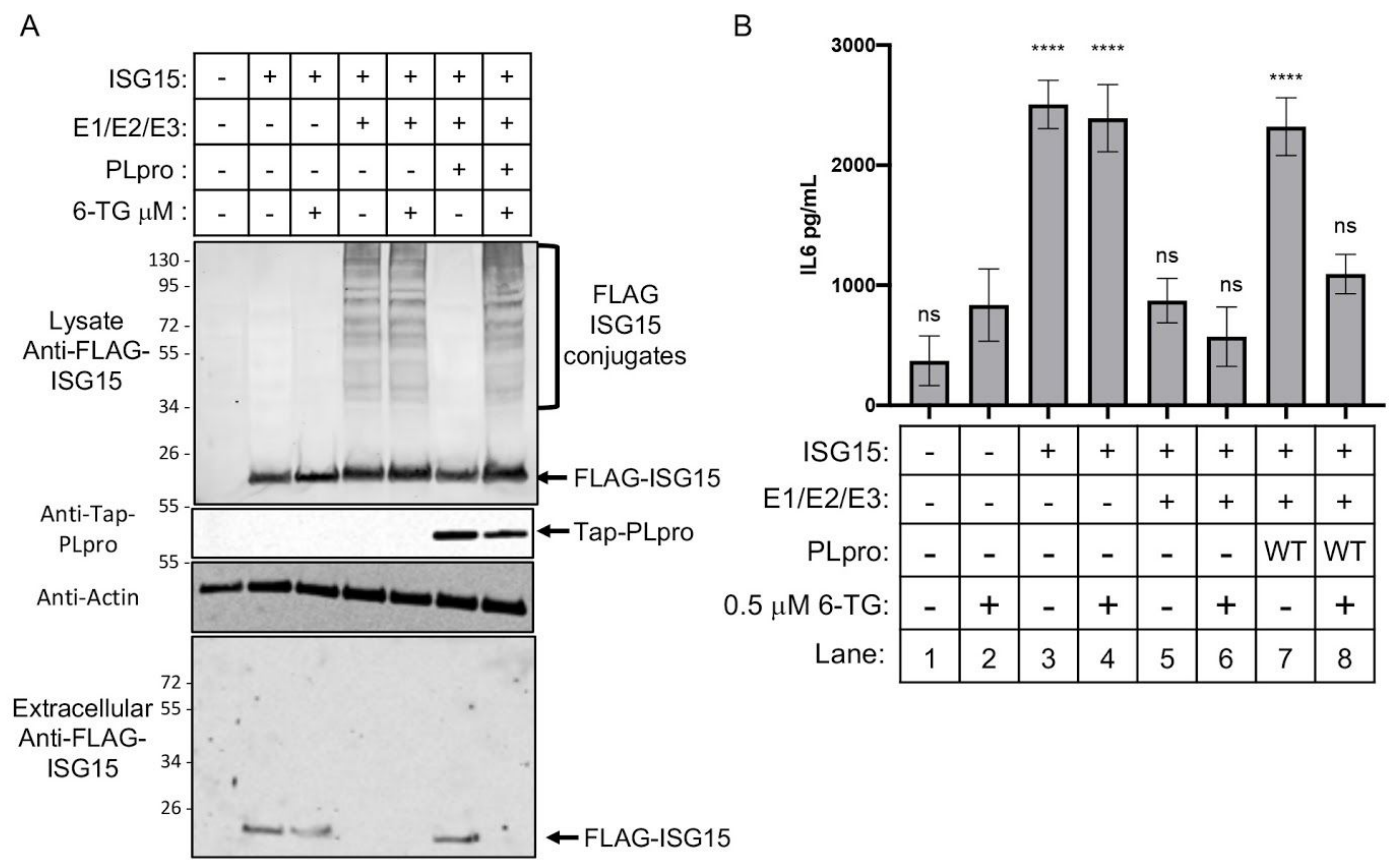

Figure 2. 6-TG inhibits PLpro-enhanced extracellular ISG15 signaling and production of IL-6 from PBMCs. A. HEK293T cells were transfected and treated with 6-TG, as indicated. Total cell lysates were prepared and cell culture supernatants were collected 48 hours post-transfection. The upper panel shows a FLAG-ISG15 immunoblot analysis of cell lysates and the lower panel shows a FLAG-ISG15 immunoblot of an anti-FLAG immunoprecipitation of the cell culture supernatants. B. HEK293T cells were plated in the lower chamber of a transwell plate and transfected with plasmids expressing FLAG-ISG15, the ISG15 E1/E2/E3 enzymes, and PLpro, as indicated. 6-TG $(0.5 \mu \mathrm{M})$ was added to the cells at the time of transfection. PMBCs were added to the upper chamber of the transwell plate; supernatants were collected from the upper chamber 24 hours post-transfection and assayed for IL-6 by ELISA. Significance was assessed by ordinary one-way ANOVA comparison to treatment with 6-TG alone. Asterisks indicate p-values: $* * * *<0.0001$, and non-significant changes are indicated by ns. 
A

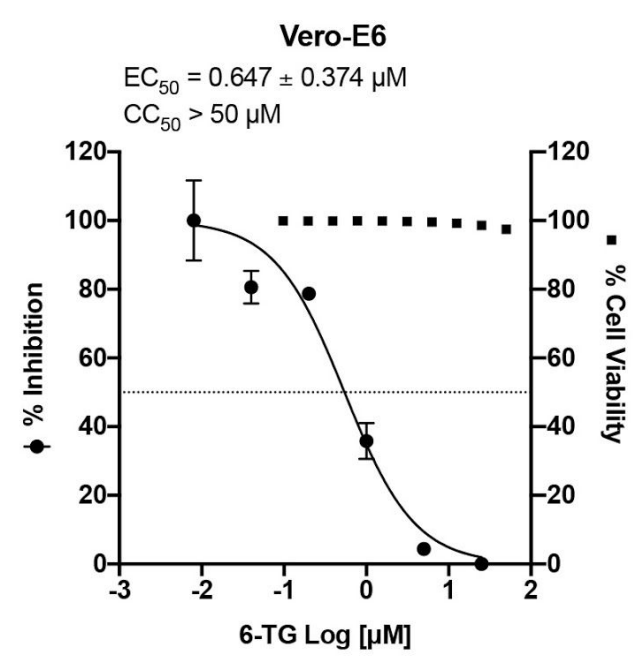

C

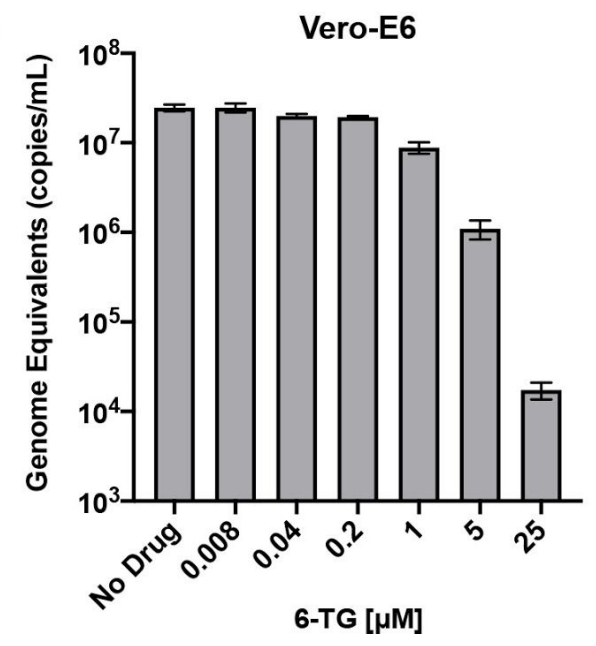

B

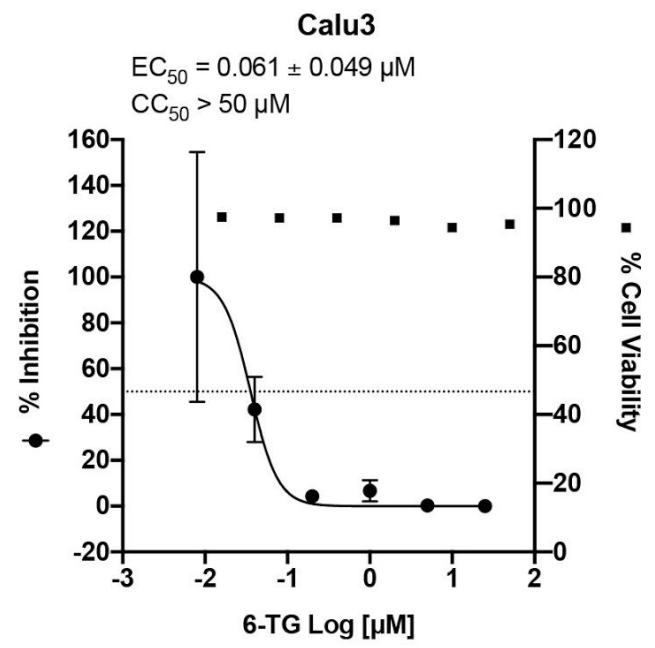

D

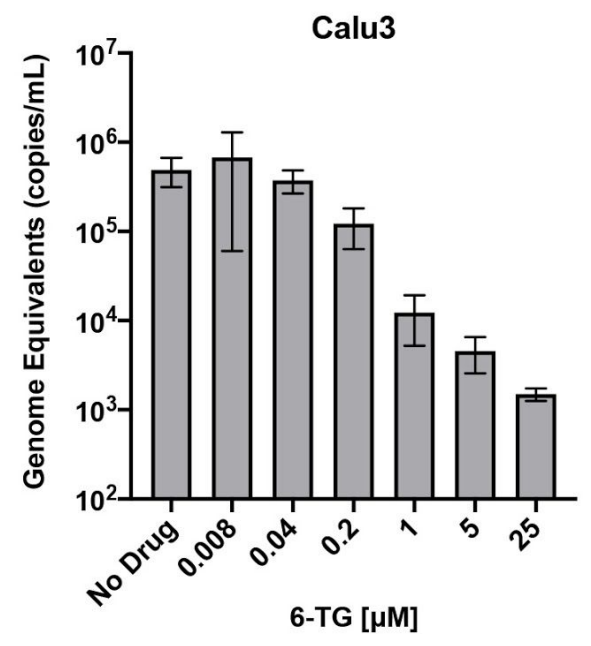

Figure 3. 6-TG inhibits SARS-CoV-2 viral replication. Vero-E6 (A, C) and Calu3 (B, D) cells were treated with various concentrations of 6-TG and assessed for viability or were infected with SARS-CoV-2 at a multiplicity of infection (MOI) of 0.001. A and B: Cellular cytotoxicity of 6-TG was determined using a real-time SYTOX assay 48 hours post-treatment (closed squares) and the median cytotoxic concentration (CC50) was $>50 \mathrm{mM}$ in both cell lines. Inhibition of viral replication was assayed by SARS-CoV-2 N1 RT-qPCR of cell supernatants 48 hours post-infection (closed circles) and the effective median concentration for inhibition (EC50) was determined. The left and right $y$-axis of each graph represent mean percent inhibition of virus yield/titer and mean percent cell viability at the indicated concentration of 6-TG. C. and D. Viral genome equivalents in the cell supernatants were quantified by SARS-CoV-2 N1 RT-qPCR. Data are means \pm SED; $\mathrm{n}=3-5$ biological replicates. 


\section{Supplementary Materials for}

\section{Title: 6-Thioguanine blocks SARS-CoV-2 replication by inhibition of PLpro protease activities}

Authors: Caleb D. Swaim ${ }^{1}$, Yi-Chieh Perng ${ }^{2}$, Xu Zhao ${ }^{1}$, Larissa A. Canadeo ${ }^{1}$, Houda H.

Harastani $^{2}$, Tamarand L. Darling ${ }^{2}$, Adrianus C. M. Boon ${ }^{2,3,4}$, Deborah J. Lenschow ${ }^{2,3}$, and Jon M. Huibregtse ${ }^{1 *}$.

Affiliations: ${ }^{1}$ Department of Molecular Biosciences, University of Texas at Austin, Austin, TX,

${ }^{2}$ Department of Internal Medicine, ${ }^{3}$ Department of Pathology and Immunology, ${ }^{4}$ Department of Molecular Microbiology, Washington University School of Medicine, St. Louis, MO.

*Correspondence to: huibregtse@austin.utexas.edu.

\section{This PDF file includes:}

Materials and Methods

Fig. S1 


\section{Materials and Methods}

\section{Cells, viruses, and compounds.}

All cell lines were maintained at $37^{\circ} \mathrm{C}, 5 \% \mathrm{v} / \mathrm{v} \mathrm{CO}$ in a humidified incubator. HEK293T cells were grown in DMEM (Corning) supplemented with 10\% FBS (Sigma) and 1\% Penicillin-Streptomycin (Corning). PBMCs from healthy individuals were isolated from Leukoreduction System (LRS) Chambers obtained from We Are Blood (Austin, TX). The LRS chamber blood was diluted 1:1 with PBS with 2\% FBS and transferred to a SepMate tube (Stem Cell Technologies) containing an equal volume of Ficoll Plaque (GE Healthcare). The tube was centrifuged at $1,200 \times \mathrm{g}$ for 10 minutes at room temperature. The top layer containing the enriched mononuclear cells (MNCs) was poured off to a separate tube. The enriched MNCs were washed twice with PBS containing 2\% FBS. To eliminate any red blood cells (RBCs) present in the MNC fraction, cells were lysed with 1X RBC lysis buffer (Biolegend). The MNCs were then counted and either frozen in RPMI 1640 containing 20\% FBS and 5\% DMSO or plated in RPMI 1640 at $1 \times 10^{6}$ cells per $\mathrm{mL}$.

Vero-E6 (ATCC), Calu3 (ATCC) were cultured at $37^{\circ} \mathrm{C}, 5 \% \mathrm{v} / \mathrm{v} \mathrm{CO}_{2}$ in a humidified incubator in Dulbecco's Modified Eagle medium (Corning) supplemented with 10\% FBS (HyClone), 1\% Penicillin-Streptomycin (Corning), 10 mM HEPES (Corning), and 1X non-essential amino acids (Sigma). Infections and culture post infection was conducted in DMEM with 2\% FBS, 1\% Penicillin-Streptomycin, 10mM HEPES, and 1X non-essential amino acids.

SARS-CoV-2 strain 2019 n-CoV/USA_WA1/2020 was originally obtained from the Centers for Disease Control and Prevention (CDC/BEI Resources NR52281) and was a gift of 
Michael S. Diamond, Washington University School of Medicine. All work with infectious SARS-CoV2 was performed in Institutional Biosafety Committee approved A-BSL3 facilities at Washington University School of Medicine using appropriate positive pressure air respirators and protective equipment.

\section{$\underline{\text { Plasmids }}$}

pcDNA3.1-TAP-PLpro $^{\mathrm{WT}}$ and pcDNA3.1-TAP-PLpro ${ }^{\mathrm{CA}}$ were described in (7). pcDNA3.1-TAP-nsp123 was generated from a partial orf1ab clone (S2-A2_p57 plasmids) encoding residues 1-1504; nsp1, nsp2, and part of nsp3; (gift from Hongbing Jiang and David Wang, Washington University School of Medicine ) with the remainder of the nsp3 ORF (residues 1505 - 2767 of pp1a, derived from Addgene cat\# 141257). The pcDNA3.1-TAP-nsp123 active site variant (C to A substitution, residue 1677 of polyprotein 1a, corresponding to residue 856 of mature nsp3) was generated from this clone by overlapping PCR. pGEX6P ISG15 was described previously (12) and the pGEX6P ISG15-precursor was made by adding nucleotides corresponding to the amino acid sequence TEPGGRS YPYDVPDYA (the natural ISG15 C-terminal extension and an HA tag) to the 3' end of pGEX6P ISG15. Plasmids expressing FLAG-ISG15, and the ISG15 E1, E2, and E3 enzymes (Uba7, Ube2L6, and Herc5) have been described previously (12).

\section{Transfections and drug treatments}

HEK293T cells were plated in a 6 well plate at a density of $3 \times 10^{4}$ cells $/ \mathrm{mL}$. The next day cells were transfected using X-tremeGENE HP DNA transfection reagent (Roche). Plasmids were transfected at the following amounts: $0.5 \mu \mathrm{g}$ FLAG-Ubiquitin, $0.35 \mu \mathrm{g}$ FLAG-ISG15, 0.25 $\mu \mathrm{g}$ Uba7, $0.25 \mu \mathrm{g}$ Ube2L6, $0.35 \mu \mathrm{g}$ HA-Herc5, $1 \mu \mathrm{g}$ TAP-nsp123 (WT or CA), $0.2 \mu \mathrm{g}$ 
TAP-PLpro $^{\mathrm{CA}}$, and TAP-PLpro ${ }^{\mathrm{WT}}(0.2,0.4,0.6,0.8$, or $1.0 \mu \mathrm{g})$. Cells were treated with 6-TG at the indicated concentrations immediately following transfection (100 mM 6-TG stock in DMSO and is diluted to working concentrations in PBS).

\section{Transwell assay and IL-6 ELISA}

HEK293T cells $\left(1 \times 10^{5}\right.$ cells per well) were plated in the lower chambers of 24-well Corning transwell plates $\left(0.4 \mu \mathrm{m}\right.$ membrane; Fisher Scientific). At the same time, PBMCs $\left(1 \times 10^{6}\right.$ cells per $\mathrm{ml}$ ) were plated in a separate $10 \mathrm{~cm}$ dish in RPMI 1640 with $10 \%$ FBS and 1\% penicillin streptomycin and allowed to rest for 16 hours. HEK293T cells were transfected with $100 \mathrm{ng}$ of plasmid expressing FLAG-ISG15, with or without ISG15 conjugation components (50 ng Ube1L, 50 ng UbcH8, 100 ng Herc5) and/or SARS-CoV2 PL pro, using X-tremeGENE HP DNA transfection reagent (Roche) and treated with and without $0.5 \mu \mathrm{M}$ 6-TG. $0.3 \mathrm{~mL}$ of PBMCs (at $1 \times 10^{6}$ per $\mathrm{mL}$ ) were added to the upper chamber of the transwell chamber, and cultured for 48 hours. Cell culture supernatants were collected from the upper transwell chamber and assayed for IL-6 production by IL-6 ELISA according to the manufacturer's protocol. (Thermo Scientific).

\section{Protein purification.}

ISG15 and ISG15-precursor (ISG15-pre) proteins were purified as GST fusion proteins in BL21 E. coli. Overnight starter cultures were grown at $37^{\circ} \mathrm{C}$ for 16 hours. Cultures were diluted 1:10 and cultured with shaking for 2 hours at $37^{\circ} \mathrm{C}$. Expression of proteins was induced with 100 $\mu \mathrm{M}$ Isopropyl $\beta$-D-1-thiogalactopyranoside (IPTG) for 3 hours at $30^{\circ} \mathrm{C}$. Cells were collected by centrifugation, resuspended in $10 \mathrm{~mL}$ PBS with $1 \%$ Triton X-100, and sonicated for 30 seconds. Lysates were spun at $15,000 x g$ for 10 minutes, and supernatants were incubated with $100 \mu \mathrm{L}$ 
Glutathione Sepharose (GE Healthcare) for 2 hours with rotation at $4^{\circ} \mathrm{C}$. Beads were collected and washed $3 \mathrm{X}$ with PBS plus $1 \%$ Triton $\mathrm{X}-100$ and $3 \mathrm{X}$ with $50 \mathrm{mM}$ Tris $\mathrm{pH} 8.0,150 \mathrm{mM} \mathrm{NaCl}$, 0.01\% Triton X-100. GST-ISG15-pre was eluted in $100 \mu \mathrm{L}$ of $10 \mathrm{mM}$ reduced GSH for $1 \mathrm{hr}$ at room temperature.

Sars-CoV-2 PLpro was purified as a GST fusion protein in BL12 E. coli. Overnight starter cultures were grown at $37^{\circ} \mathrm{C}$ for 16 hours. Cultures were diluted 1:10 and cultured with shaking for 2 hours at $37^{\circ} \mathrm{C}$. Expression of proteins was induced with $100 \mu \mathrm{M}$ Isopropyl $\beta$-D-1-thiogalactopyranoside (IPTG) for 24 hours at $16^{\circ} \mathrm{C}$. Cells were collected by centrifugation, resuspended in $10 \mathrm{~mL}$ TBS with $0.00025 \%$ Tween $20,0.01 \mathrm{mM}$ DTT and 5\% glycerol (Binding Buffer), and sonicated for 1 minute. Lysates were spun at 15,000xg for 10 minutes and supernatants were incubated with $100 \mu \mathrm{L}$ per liter of culture Glutathione Sepharose (GE Healthcare) for 4 hours with rotation at $4^{\circ} \mathrm{C}$. Beads were collected and washed three times with Binding Buffer supplemented with 0.1mM EDTA and subjected to site-specific cleavage with PreScission Protease (GE Healthcare) to remove the GST tag. Beads were removed and the protein concentration in the supernatant was quantified by SDS-PAGE using a Licor Odyssey Imager.

\section{In vitro cleavage of GST-ISG15-pre.}

$0.1 \mu \mathrm{M}$ PLpro was incubated with $0.1 \mu \mathrm{M}$ GST-ISG15-pre for the indicated amount of time with the indicated amount of 6-TG. Reactions were initiated by the addition of GST-ISG15-pre and stopped by addition of $10 \mu \mathrm{L}$ of $250 \mathrm{mM}$ tris $\mathrm{pH} 6.8,40 \%$ glycerol $8 \%$ SDS and 400mM DTT. Samples were run on 10\% Tris-Glycine gels and stained with Coomassie blue stain. Gels were imaged using a Licor Odyssey Imager. 


\section{Immunoblotting and immunoprecipitations}

Samples assessing ISGylation or ubiquitination were lysed in 1\% NP40 lysis buffer (100 mM Tris $\mathrm{pH} 7.5,100 \mathrm{mM} \mathrm{NaCl}, 1 \%$ NP40) supplemented with $10 \mathrm{mM}$ NEM, $170 \mu \mathrm{g} / \mathrm{mL}$ PMSF, $2 \mu \mathrm{g} / \mathrm{mL}$ leupeptin, $2 \mu \mathrm{g} / \mathrm{mL}$ aprotinin and $10 \mathrm{mM}$ DTT for 10 minutes on ice followed by a 10 minute spin at $20000 \times \mathrm{g}$. Samples were run on BOLT 4-12\% Bis-Tris gels (Thermo) and blotted with the M2 FLAG antibody (Sigma). Samples assessing the effect of 6-TG on TAP-nsp123 cleavage were lysed in RIPA buffer (50 mM Tris $\mathrm{pH} 8,150 \mathrm{mM} \mathrm{NaCl}, 0.5 \%$ NP40, 0.1\% SDS, 0.5\% Sodium deoxycholate w/v) supplemented with $10 \mathrm{mM} \mathrm{NEM,} 170 \mu \mathrm{g} / \mathrm{mL}$ PMSF, $2 \mu \mathrm{g} / \mathrm{mL}$ leupeptin, $2 \mu \mathrm{g} / \mathrm{mL}$ aprotinin, $10 \mathrm{mM}$ DTT, and 2 units/mL Universal Nuclease (Pierce) for 10 minutes on ice followed by a 10 minute spin at $20000 \mathrm{x}$. Samples were run on $3-8 \%$ Tris-Acetate gels (Thermo) and blotted for TAP using the anti-protein A antibody (Sigma). Actin blots were performed with an anti actin antibody clone: AC-15 (Invitrogen).

For detection of extracellular ISG15, cell culture supernatants were collected and cleared of any cell debris by centrifugation at $1000 \times \mathrm{g}$. The supernatants were further cleared of $\operatorname{IgG}$ from the serum by two one-hour bindings with Protein A sepharose (Invitrogen) at room temperature. $20 \mu \mathrm{L}$ of Sera-Mag SpeedBead Protein A/G magnetic beads (GE Life Sciences), 10 $\mu \mathrm{L}$ of ISG15 antibody (Invitrogen 7H29L24) and $20 \mathrm{~mL}$ of $0.1 \%$ NP40 in PBS pH 7.0 were added to the cell culture supernatants and rocked for 2 hours at room temperature. Beads were isolated with a neodymium magnet and washed $3 \mathrm{X}$ with $0.1 \%$ NP40 in PBS pH 7.0. ISG15 was eluted from the beads by boiling in $80 \mu \mathrm{l}$ of $1 \mathrm{X}$ loading buffer and $40 \mu \mathrm{l}$ was run on a NuPAGE 4-12\% Bis-Tris gel and transferred to nitrocellulose for western blotting. ISG15 was detected with the M2 Flag antibody (Sigma). 


\section{Virus replication assays}

1.5-2 x $10^{5}$ Vero-E6 and $5 \times 10^{5}$ Calu3 cells were seeded in a 24 well format. The cells were infected with SARS-CoV-2 at a MOI of 0.001 diluted in culture media containing 2\% FBS. Virus inoculum was removed after one hour and cells were washed with $1 \mathrm{~mL}$ PBS twice. Infected cells were cultured in media containing 6-TG $(25 \mu \mathrm{M}, 5 \mu \mathrm{M}, 1 \mu \mathrm{M}, 200 \mathrm{nM}, 40 \mathrm{nM}$, 8nM, NoTx). $100 \mu \mathrm{l}$ of culture supernatants were harvested into (ORF) $300 \mu \mathrm{L}$ TRK lysis buffer plus BME at 24 and 48 hours. RNA was isolated from homogenates of TRK lysates using an E.Z.N.A. Total RNA kit I (Omega). Isolated RNA was resuspended in UltraPure $\mathrm{H}_{2} \mathrm{O}$ (Ambion). RT-qPCR was performed on a ViiA 7 or an ABI 7500 qPCR machine (Thermo Scientific) using the TaqMan RNA-to-CT 1-Step Kit (Applied Biosystems) with a $20 \mu \mathrm{L}$ total reaction volume per well including $8.5 \mu \mathrm{L}$ of RNA sample and Taqman primers and SARS-CoV2 N1 probe of 2019-nCoV RUO Kit from Integrated DNA Technologies. The following PCR cycling protocol was used: 15 minutes at $48^{\circ}, 10 \mathrm{~min}$ at $95^{\circ}$, and 50 cycles of 15 sec at $95^{\circ}$ and 1 min at $60^{\circ} \mathrm{C}$. RNA copy number was determined for each sample using the matching RNA standard (gift of Adam Bailey, Michael S. Diamond lab, Washington University School of Medicine).

\section{6-TG toxicity assays}

The real-time cytotoxicity events were analyzed by using a 2-color IncuCyte ZOOM in-incubator imaging system (Essen Bioscience). Dead cells were detected by uptake of the cell-impermeable dye SYTOX Green (Invitrogen). The total cell numbers were estimated by calculating cell numbers staining with cell-permeable dye SYTO24. 


\section{Quantification and Statistical analysis.}

Graphpad Prism 8 software was used to plot ELISA, virus replication and densitometry data. Bars represent three or more biological replicates. Error bars represent the standard error of the mean (SEM). Ordinary one-way ANOVA was performed on each ELISA data set comparing all conditions to treatment with 6-TG, alone. $\mathrm{EC}_{50}$ and $\mathrm{CC}_{50}$ of 6-TG were calculated using "log(inhibitor) vs. normalized response -- Variable slope". Cell viability, dose response curve, and $\mathrm{EC}_{50}$ with $95 \%$ confidence interval was plotted/indicated in the graph. Asterisks indicate

p-values: $*=0.02, * *=0.002, * * *=0.004, * * * *<0.0001$, and non significant changes are indicated by ns. 

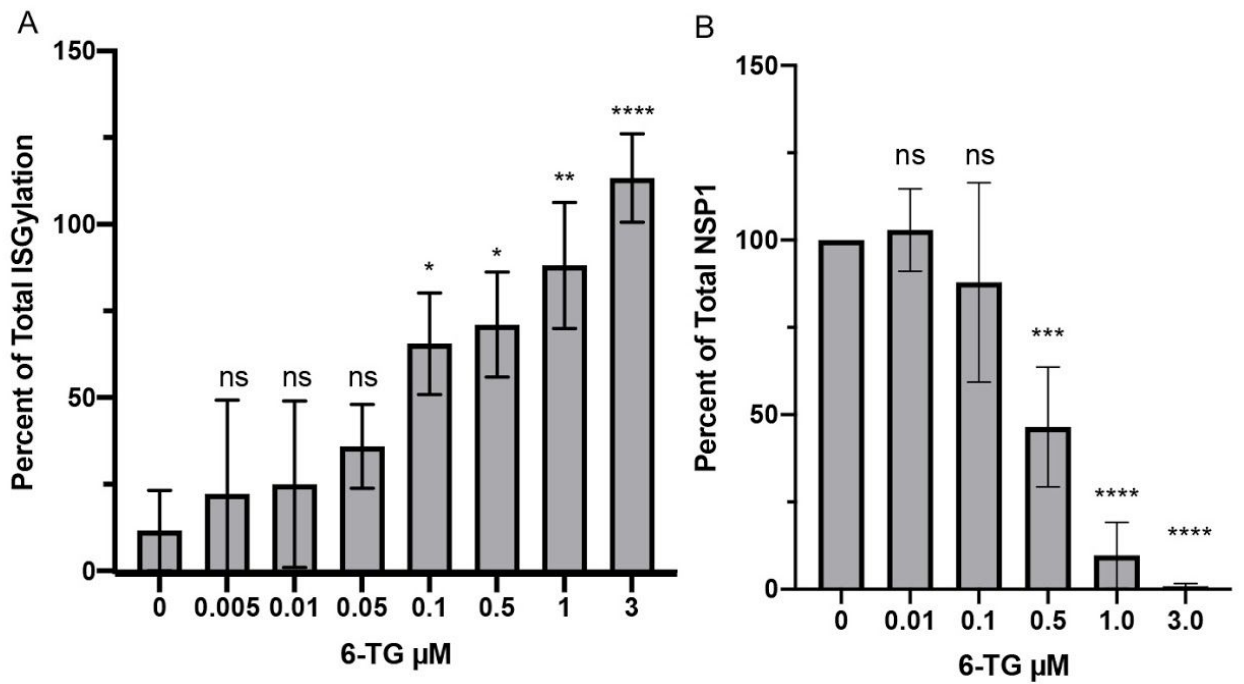

Fig. S1. Quantification of ISGylation and nsp123 cleavage by PLpro. A. Densitometry analysis of ISGylation from Fig. 1A. Percent of total ISGylation was determined by comparison to total ISGylation in the presence of PLpro ${ }^{\mathrm{CA}}$. B. Densitometry analysis of TAP-nsp1 from Figure 1B. Percent TAP-nsp1 was determined by comparison to the $42 \mathrm{kDa}$ band in the untreated samples to those present in samples treated with 6-TG. Significance was assessed by ordinary one-way ANOVA comparison to treatment with no 6-TG. Asterisks indicate p-values: $*=0.02$, $* *=0.002, * * *=0.004, * * * *<0.0001$, and non-significant changes are indicated by ns. 